\title{
Measuring the stellar initial mass function
}

\author{
Andrew Hopkins
}

AAO/Macquarie University, Australia

\begin{abstract}
The birth of stars and the formation of galaxies are cornerstones of modern astrophysics. While much is known about how galaxies globally and their stars individually form and evolve, one fundamental property that affects both remains elusive. This is problematic because this key property, the stellar initial mass function (IMF), is a key tracer of the physics of star formation that underpins almost all of the unknowns in galaxy and stellar evolution. It is perhaps the greatest source of systematic uncertainty in star and galaxy evolution. The past decade has seen a growing number and variety of methods for measuring or inferring the shape of the IMF, along with progressively more detailed simulations, paralleled by refinements in the way the concept of the IMF is applied or conceptualised on different physical scales. This range of approaches and evolving definitions of the quantity being measured has in turn led to conflicting conclusions regarding whether or not the IMF is universal. Here I summarise the growing wealth of approaches to our understanding of this fundamental property that defines so much of astrophysics, and highlight the importance of considering potential IMF variations, reinforcing the need for measurements to quantify their scope and uncertainties carefully. I present a new framework to aid the discussion of the IMF and promote clarity in the further development of this fundamental field.
\end{abstract}

